\title{
Recent results from the CBELSA/TAPS experiment at ELSA
}

\author{
Farah Afzal for the CBELSA/TAPS collaboration ${ }^{1, *}$ \\ ${ }^{1}$ Helmholtz-Institut für Strahlen- und Kernphysik, Universität Bonn, Germany
}

\begin{abstract}
In order to gain a better understanding of the dynamics inside the nucleon and of the non-perturbative regime of QCD, the nucleon excitation spectra and the properties of nucleon resonances are investigated. An essential experimental tool to achieve this goal is the study of different photoproduction reactions. Partial wave analyses are performed in order to obtain information about the contributing resonances. A complete experiment is needed to extract the underlying amplitudes unambiguously, which requires the measurement of carefully chosen single and double polarization observables in addition to the unpolarized cross section. The CBELSA/TAPS experiment in Bonn offers the possibility to measure several polarization observables using a linearly or circularly polarized photon beam and with a longitudinally or transversely polarized target. This contribution gives an overview of recently measured polarization observables in different final states. The impact of the new data is discussed.
\end{abstract}

\section{Introduction}

Comparisons of the nucleon or delta excitation spectra to either quark model predictions [1] or latest lattice QCD calculations [2] reveal that the dynamics inside the nucleons are not well understood in the non-perturbative regime of QCD. In particular, more states are predicted than there have been experimentally observed so far. This feature is known as the missing resonances problem. One possible explanation for this could be that most of the states, which were found in the past, were observed in $\pi N$ scattering experiments. In case some resonances may not couple strongly to the $\pi N$, they could have been missed. Therefore, it is crucial to probe the excitation spectra using different production mechanisms such as the photoproduction of mesons. Several facilities around the world have been studying photoproduction reactions like the CLAS collaboration at JLab [3-5], the A2 collaboration at MAMI [6], the LEPS collaboration at SPring-8 [7] and the CBELSA/TAPS collaboration at ELSA [8-14].

The determination of the resonance parameters from the data is a complicated task since the resonances are broad and overlap strongly. Thus, partial wave analyses (PWA) are necessary to disentangle the contributing resonances and their parameters. Different PWA approaches exist e.g. the $\mathrm{BnGa}$ [15], the JüBo [16], the SAID [17] or MAID [18] PWA. In order to obtain a unique solution, it is not sufficient to only measure the total unpolarized cross section as it is only sensitive to the squared moduli of the partial wave amplitudes. Using a polarized photon beam, a polarized target or by measuring the recoil nucleon polarization, it is possible to measure different polarization observables. Table 1

*e-mail: afzal@hiskp.uni-bonn.de shows all the possible combinations of single and double polarization observables that can be measured in the photoproduction of single pseudoscalar mesons. These polarization observables have a sensitivity to interference terms between different partial waves. Therefore, the polarization observables enhance the possibility to detect contributions from small partial waves, via interference of the small waves with large dominant partial waves.

For more than a decade the main focus of the CBELSA/TAPS experiment in Bonn has been dedicated to acquiring polarization observables for different final states. This contribution reports on the latest measured data and their impact.

\section{Experimental setup}

The ELectron Stretcher Accelerator (ELSA) [19] provides unpolarized or longitudinally polarized electrons with an energy of up to $3.2 \mathrm{GeV}$. The electron beam is delivered either to the BGO-OD [20] or to the CBELSA/TAPS experimental area (see Figure 1). The electrons impinge on a radiator located inside the goniometer tank and produce a photon beam via bremsstrahlung. The scattered electrons are deflected according to their momenta in the magnetic field of the tagging system and detected by scintillation fibers and bars.

A thin diamond crystal is used as radiator in order to have coherent bremsstrahlung. The diamond crystal lattice can be positioned precisely with respect to the electron beam, which allows to control the coherent edge position [21]. A maximal polarization degree of $65 \%$ is achieved for a coherent edge position of $E_{\gamma}=950 \mathrm{MeV}$ and of $35 \%$ for $E_{\gamma}=1850 \mathrm{MeV}$. Longitudinally polarized electrons in combination with an amorphous radiator are utilized to get 
Table 1. Table lists all possible polarization observables that can be obtained by using a polarized photon beam, a polarized target by measuring the recoil nucleon polarization degree. Some observables can be measured in two different ways, e.g. $P$ can be measured as a single polarization observable or as a double polarization observable with linearly polarized photon beam and a transversely polarized target. All information are taken from [22].

\begin{tabular}{l|c|ccc|cccc|cccc}
\hline \multicolumn{1}{l}{ photon beam } & \multicolumn{3}{c}{ target } & \multicolumn{4}{c}{ recoil nucleon } & \multicolumn{3}{c}{ target and recoil } \\
\hline & & $\mathrm{x}$ & $\mathrm{y}$ & $\mathrm{z}$ & - & - & - & $\mathrm{x}$ & $\mathrm{z}$ & $\mathrm{x}$ & $\mathrm{z}$ \\
& & - & - & - & $\mathrm{x}$ & $\mathrm{y}$ & $\mathrm{z}$ & $\mathrm{x}$ & $\mathrm{x}$ & $\mathrm{z}$ & $\mathrm{z}$ \\
\hline- & $\sigma_{0}$ & - & $T$ & - & - & $P$ & - & $T_{x^{\prime}}$ & $L_{x^{\prime}}$ & $T_{z^{\prime}}$ & $L_{z^{\prime}}$ \\
linear & $-\Sigma$ & $H$ & $(-P)$ & $G$ & $O_{x^{\prime}}$ & $(-T)$ & $O_{z^{\prime}}$ & $\left(-L_{z^{\prime}}\right)$ & $\left(T_{z^{\prime}}\right)$ & $\left(L_{x^{\prime}}\right)$ & $\left(-T_{x^{\prime}}\right)$ \\
circular & & $F$ & - & $-E$ & $C_{x^{\prime}}$ & - & $C_{z^{\prime}}$ & - & - & - & - \\
\hline
\end{tabular}

circularly polarized photons. Here, a maximal polarization degree of around $65 \%$ could be reached for the maximal beam photon energy that is possible to tag for an ELSA energy of $E_{0}=2400 \mathrm{MeV}$.

The photons impinge a target which is located in the center of the Crystal Barrel (CB) calorimeter [23]. As target material an unpolarized liquid hydrogen is used to perform measurements on free protons. A longitudinally or transversely polarizable frozen-spin butanol or deuterized butanol target is available for the measurement of polarization observables with polarized protons or neutrons, respectively [24]. A maximal polarization degree of $p_{T}=84 \%$ and high relaxation times of around 2000 $\mathrm{h}$ were reported for the transversely polarized butanol target of most recent beamtimes, which has been possible due to a collaborative effort between the target group of the CBELSA/TAPS collaboration in Bonn and the target group of the A2 collaboration in Mainz [25]. In addition, a carbon foam target is used to study the unpolarized background contribution of the butanol target. Furthermore, inmedium modification of mesons are investigated by utilizing different solid targets.

The CB calorimeter consists of $1320 \mathrm{CsI}(\mathrm{Tl})$ crystals that cover a large polar angular range from $30^{\circ}$ to $156^{\circ}$ and the full azimuthal angular range. Each crystal is now read out by two avalanche photodiodes instead of the previously used PIN photodiodes. The new readout electronics allows to use the $\mathrm{CB}$ in the first level trigger which is crucial for a high trigger efficiency of complete neutral final states, e.g. $n \pi^{0}$. The upgrade was successfully completed and new data were taken since December 2017.

In forward direction, the MiniTAPS calorimeter [26] is located, which is made of $216 \mathrm{BaF}_{2}$ crystals and readout by photomultipliers in order to gain a fast trigger signal.

A cylindrically formed scintillation fiber detector [27] is located around the target. Together with scintillation plates, that are present in front of all the crystals that fulfill $\theta<30^{\circ}$, the identification of charged particles is possible.

\subsection{Determination of polarization observables}

Since the CBELSA/TAPS experiment is equipped with two electromagnetic calorimeters, it is ideally suited for detecting photons in the final state. Thus, this experiment focuses on reconstructing neutral mesons that decay into two or more photons. Figure 2 shows a typical two photon invariant mass spectrum. It is possible to obtain high statistics data sets with very low background contributions for single meson final states like the $p \pi^{0}$ and $p \eta$ final states. In addition, final states like $p \omega$ or $p \eta^{\prime}$ can be selected as well. Furthermore, multi-meson final states like $p \pi^{0} \pi^{0}$ or $p \pi^{0} \eta$ have been successfully selected.

As already mentioned, the CBELSA/TAPS experiment provides the possibility to measure with a polarized photon beam and with a polarized target. Thus, the CBELSA/TAPS setup can be used to measure the polarization observables of the beam-target category (see Table $1)$. The polarized cross section $\frac{d \sigma}{d \Omega}$ pol for a single pseudoscalar meson reads [22]

$$
\begin{aligned}
\frac{d \sigma}{d \Omega_{\mathrm{pol}}} & =\frac{d \sigma}{d \Omega_{0}}\left[1-p_{\gamma}^{\operatorname{lin}} \boldsymbol{\Sigma} \cos (2 \varphi)\right. \\
& +p_{x}\left(p_{\gamma}^{\operatorname{lin}} \mathbf{H} \sin (2 \varphi)+p_{\gamma}^{\mathrm{circ}} \mathbf{F}\right) \\
& -p_{y}\left(p_{\gamma}^{\operatorname{lin}} \mathbf{P} \cos (2 \varphi)-\mathbf{T}\right) \\
& \left.-p_{z}\left(-p_{\gamma}^{\operatorname{lin}} \mathbf{G} \sin (2 \varphi)+p_{\gamma}^{\operatorname{circ}} \mathbf{E}\right)\right],
\end{aligned}
$$

where $p_{\gamma}^{\text {lin }}$ and $p_{\gamma}^{\text {circ }}$ are the degree of linearly and circularly polarized photons, $p_{x}, p_{y}$ and $p_{z}$ give the target polarization degree in transverse and longitudinal direction and $\frac{d \sigma}{d \Omega}$ is the unpolarized differential cross section.

For the measurement of the beam asymmetry $\Sigma$ data were taken with a linearly polarized photon beam and a liquid hydrogen target in 2013. Here, the coherent edge settings were chosen at high beam photon energies of $E_{\gamma}=$ $1750 \mathrm{MeV}$ and $E_{\gamma}=1850 \mathrm{MeV}$, where high precision data is scarce for many final states.

Using a polarized frozen-spin butanol target instead of the hydrogen target, allows to measure the double polarization observable $H$. In addition, the polarization observables $T$ and $P$, whereby $P$ is measured here as a double polarization observable (see Table 1), can be accessed simultaneously as well. Data were taken with this configuration before and after the CBELSA/TAPS upgrade using different coherent edge settings (from $E_{\gamma}=950 \mathrm{MeV}$ to $E_{\gamma}=$ $1300 \mathrm{MeV})[10,11]$. Furthermore, the double polarization observable $G$ was measured in a separate beam time using a longitudinally polarized butanol target in combination with linearly polarized photons $[12,13]$. Besides, the 


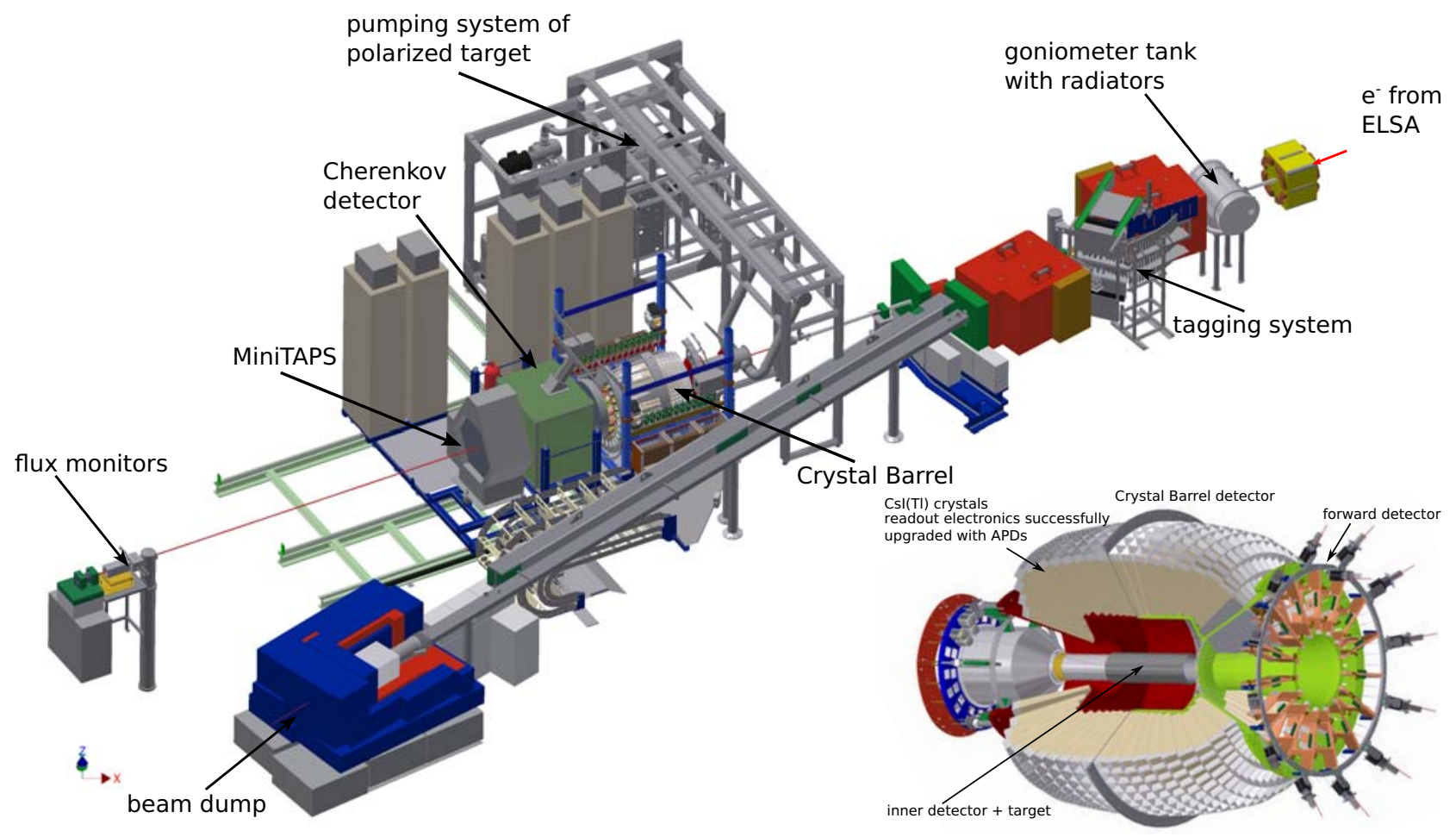

Figure 1. Overview of the CBELSA/TPAS experimental area. The electron beam from ELSA (upper right corner) impinges a thin radiator inside the goniometer tank and a photon beam is produced via bremsstrahlung. The photon energy can be determined with the tagging system that deflects the scattered electrons according to their momenta and detects them via scintillation fibers and bars. The photons hit a target at the center of the Crystal Barrel calorimeter, which is shown in large in the right bottom corner. It is complemented by the MiniTAPS detector in forward direction. The inner detector, which consists of scintillation fibers, and the scintillation plates, that are mounted in front of the forward crystals, are utilized for the identification of charged particles.

helicity asymmetry $E$ can be accessed by using circularly polarized photons and a longitudinally polarized butanol target $[8,9]$. It is defined as [22]

$$
E=\frac{\sigma_{1 / 2}-\sigma_{3 / 2}}{\sigma_{1 / 2}+\sigma_{3 / 2}}
$$

Here, $\sigma_{1 / 2}$ and $\sigma_{3 / 2}$ are the spin dependent cross sections for antiparallel or parallel spin configuration of the incoming beam photon and target, respectively.

A complication due to the used butanol target is that it does not only contain contributions from the polarizable free hydrogen nuclei but also from the unpolarized bound carbon and oxygen nuclei. The carbon background was studied in detail using additional measurements with a carbon foam target. This allowed the determination of the so-called dilution factor, which gives the amount of polarizable hydrogen nuclei of the selected data. It is given by

$$
d=1-s_{c} \frac{N_{C}}{N_{B}},
$$

where $N_{C}$ and $N_{B}$ are the count rates of the selected carbon and butanol data, respectively and $s_{C}$ is a scaling factor that is needed to account mainly for the differences in the photon fluxes during the measurements performed with the carbon and the butanol target. References $[9,11$,
13] demonstrate in detail how the dilution factor can be determined from the data.

\subsection{Recent results}

The CBELSA/TAPS collaboration has measured several polarization observables for the photoproduction reactions $\gamma p \rightarrow p \pi^{0}$ [8-13], $\gamma p \rightarrow p \eta$ [14], $\gamma p \rightarrow p \eta^{\prime}$ [28], $\gamma p \rightarrow$ $p \pi^{0} \pi^{0}[29,30], \gamma p \rightarrow p \pi^{0} \eta[31]$ and $\gamma p \rightarrow p \omega$ [32]. Some of the recently obtained results will be discussed in the following.

\subsubsection{Single meson photoproduction: $\gamma p \rightarrow p \pi^{0}$}

The $p \pi^{0}$ channel is the most intensely studied photoproduction final state due to its large cross section. It has the largest database for the unpolarized cross section and polarization observables available now, to which the CBELSA/TAPS collaboration contributed significantly.

Figure 3 depicts preliminary results for the beam asymmetry $\Sigma$ as a function of $\cos \theta_{\pi^{0}}$ covering a large energy range from $E_{\gamma}=1131 \mathrm{MeV}$ to $E_{\gamma}=1779 \mathrm{MeV}$. The new data is in good agreement with previously measured data of the GRAAL, CLAS and LEPS collaborations. A Legendre moment analysis revealed sensitivity of this 


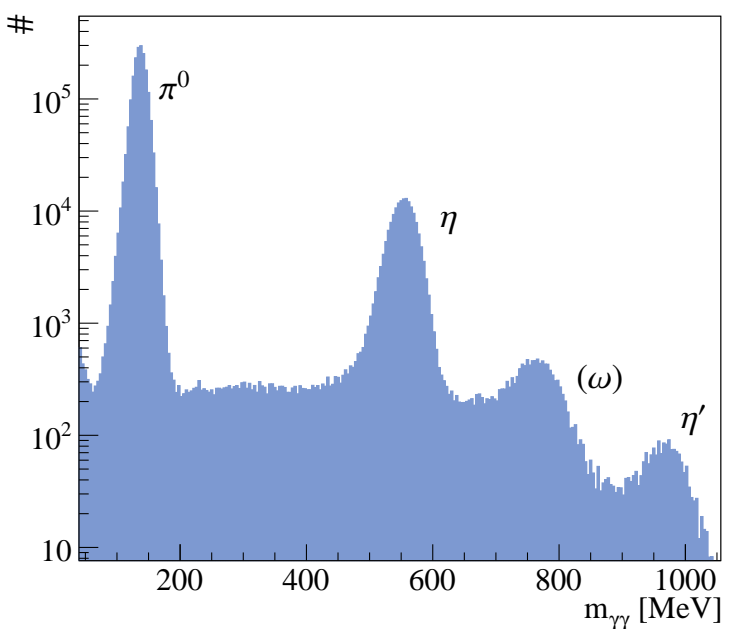

Figure 2. A typical two photon invariant mass is plotted. Clear peaks are visible for the $\pi^{0}, \eta,(\omega)$ and $\eta^{\prime}$ mesons. The background is very low for the $p \pi^{0}$ and $p \eta$ final states. The peak of the $\omega$ meson appears here due to a lost photon since it can decay to $\omega \rightarrow \pi^{0} \gamma[33]$.

and the CLAS data up to the $G$-waves [34]. This high sensitivity can only be achieved through the high precision of the data and more importantly due to the full angular coverage of the data. Latest fits from the BnGa and JüBo PWA describe the data well. Within the BnGa PWA evidence was found for the $\Delta(2200) \frac{7}{2}^{-}\left(G_{37}\right)$ resonance which can not be the parity partner of the $\Delta(1950) \frac{7}{2}^{+}\left(F_{37}\right)$ resonance due to its much higher mass [35].

The helicity asymmetry $E$ was determined for a large energy range from $E_{\gamma}=600 \mathrm{MeV}$ to $E_{\gamma}=2300 \mathrm{MeV}$ and used to determine the spin dependent total cross sections $\sigma_{1 / 2}$ and $\sigma_{3 / 2}[8,9]$, which are shown in Figure 4. It is remarkable that already in the second resonance region $\left(E_{\gamma}=660 \mathrm{MeV}\right.$ to $\left.E_{\gamma}=900 \mathrm{MeV}\right)$ discrepancies exist between data and PWA predictions. These differences could be traced back to the $N(1535) \frac{1}{2}^{-}\left(S_{11}\right)$ resonance contribution, which was underestimated by the MAID and SAID-SN11 PWA. Instead both PWAs overestimated the $N(1520) \frac{3}{2}^{-}\left(D_{13}\right)$ contribution to the $p \pi^{0}$ final state. The third resonance region $\left(E_{\gamma}=900 \mathrm{MeV}\right.$ to $E_{\gamma}=1200$ $\mathrm{MeV}$ ) is also not well described by the different PWA predictions: while the MAID, SAID-SN11 and BnGa2011-02 PWA underestimate the $\sigma_{1 / 2}$ contribution, the JüBo group overestimates it. The description of the data was significantly improved by new fits of the PWA groups.

The results for the polarization observables $T, P$ and $H$ are shown in Figure $5[10,11]$. The data are in good agreement with previously existing data and exceed them in terms of statistics, angular and energy coverage. Furthermore, the observable $H$ has been measured for the first time. The data show a relatively good agreement also to different PWA predictions.

After the CB upgrade finished, new data were taken for
$T, P$ and $H$, which are depicted in Figures 6 and 7. The new preliminary data shows a very good agreement to the already published CBELSA/TAPS data. In addition, an overall improvement of the statistical precision could be achieved by a factor of approximately two. Furthermore, the existing database for the polarization observables $P$ and $H$ could be extended to $E_{\gamma}=1300 \mathrm{MeV}$.

\subsubsection{Single meson photoproduction: $\gamma p \rightarrow p \eta$}

The database of the $p \eta$ final state is not well established in comparison to the $p \pi^{0}$ final state since the $p \eta$ cross section is much smaller. Therefore, only a scarce energy and angular coverage exists with larger statistical error bars. Nevertheless, it is prudent to study the $p \eta$ final state since the $\eta$ has an isospin of $I=0$ and can only couple to $N^{*}$ resonances and therefore provides an isospin filter.

The polarization observables $E, G, T, P$ and $H$ have been determined by the CBELSA/TAPS collaboration for the $p \eta$ final state as well. Figure 8 shows the results for the double polarization observable $G$. This new data provides much needed constraints to the $p \eta$ photoproduction amplitudes since none of the PWAs could predict the angular dependence of $G$. A new fit of the $\mathrm{BnGa}$ PWA including this new data resulted in the precise determination of $N^{*} \rightarrow p \eta$ branching ratios [14]. Here, a remarkable result is the new determined branching ratio of $0.33 \pm 0.04$ for the $N(1650) \frac{1}{2}^{-}\left(S_{11}\right)$ resonance [14]. This branching ratio was listed as $0.14-0.22$ [33] in the past and thus, was significantly smaller than the branching ratio for the $N(1535) \frac{1}{2}^{-}\left(S_{11}\right)$ with $0.42_{-0.12}^{+0.13}$ [33], which was topic of intense discussions [36].

The beam asymmetry $\Sigma$ was determined for the $p \eta$ final state as well, providing a precise data-set with a large energy and full angular coverage. A Legendre moment analysis was performed here as well, which showed evidence for the $p \eta^{\prime}$-cusp in the $E_{0+}$ multipole of $p \eta[37,38]$. Thus, the unprecedented precision in the new beam asymmetry data puts a reinforced requirement on the PWA groups to implement all relevant singularities correctly into their amplitude parametrizations.

\subsubsection{Double meson photoproduction: $\gamma p \rightarrow p \pi^{0} \pi^{0}$}

Multi-meson photoproduction final states, like the $p \pi^{0} \pi^{0}$ final state, play an important role at high center-of-mass energies, where cascading decays can be observed. Figure 9 shows a Dalitz plot for the $p \pi^{0} \pi^{0}$ final state. The visible horizontal and vertical bands indicate the intermediate resonances $\left(\Delta(1232) \frac{3}{2}^{+}, N(1520) \frac{3}{2}^{-}\right.$and $\left.N(1680) \frac{5}{2}^{+}\right)$ over which the cascading decays can occur. In addition, studying the $p \pi^{0} \pi^{0}$ final state has the advantage of having fewer non-resonant contributions in comparison to the double charged pion channel.

Within the CBELSA/TAPS collaboration the $p \pi^{0} \pi^{0}$ final state has been analyzed utilizing the full three 


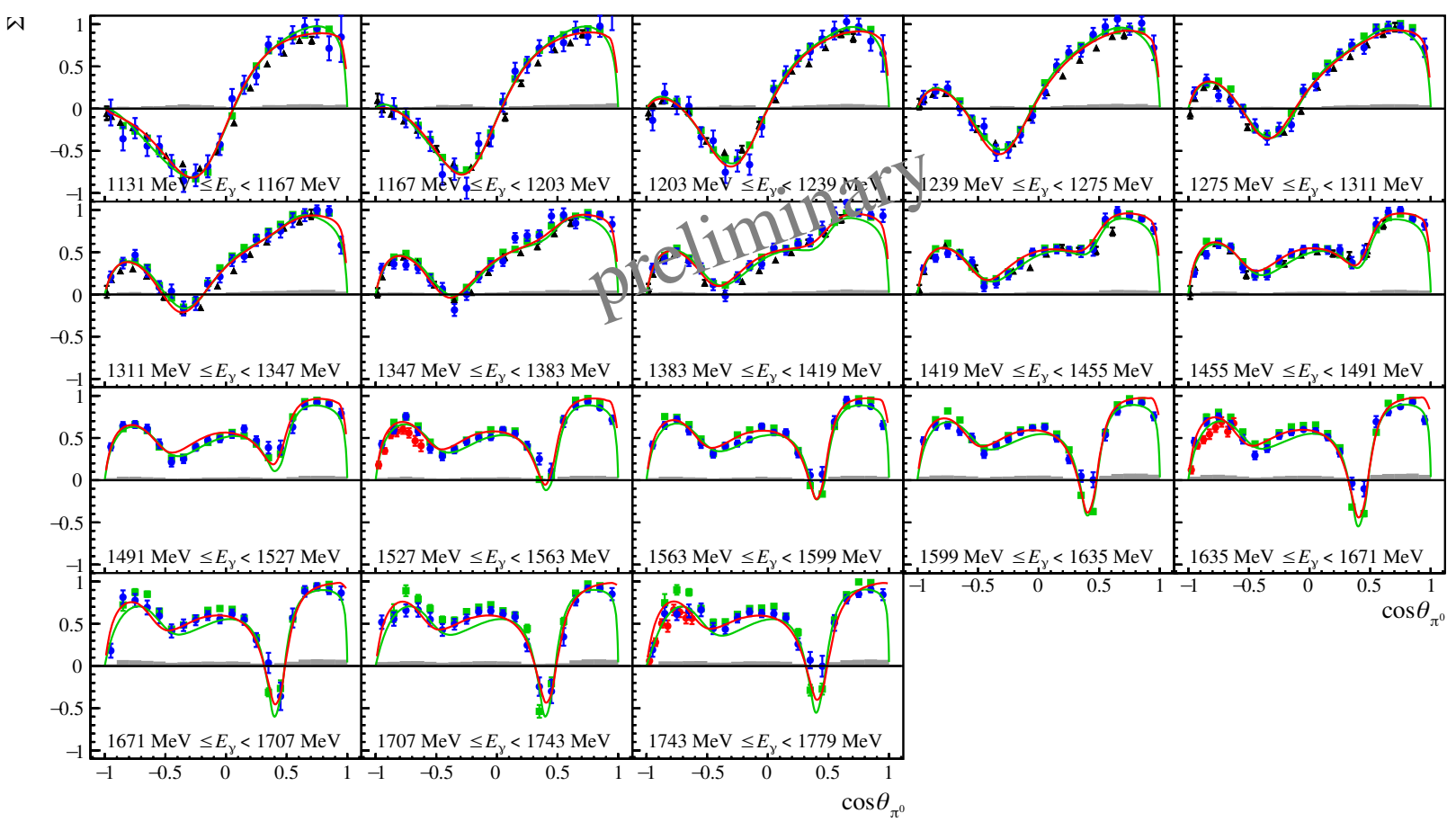

Figure 3. The preliminary results for the beam asymmetry $\Sigma$ (blue points) are shown as a function of $\cos \theta_{\pi^{0}}$ for a beam photon energy range from $E_{\gamma}=1131 \mathrm{MeV}$ to $E_{\gamma}=1779 \mathrm{MeV}$ for the $p \pi^{0}$ final state. The data are compared to data from the CLAS collaboration (green squares) [5], from the GRAAL collaboration (black triangles) [39] and from the LEPS collaboration (red points) [7]. In addition the data are compared to the latest PWA solutions of BnGa [40] and JüBo-2017 [16]. The systematic error is represented by the gray area.
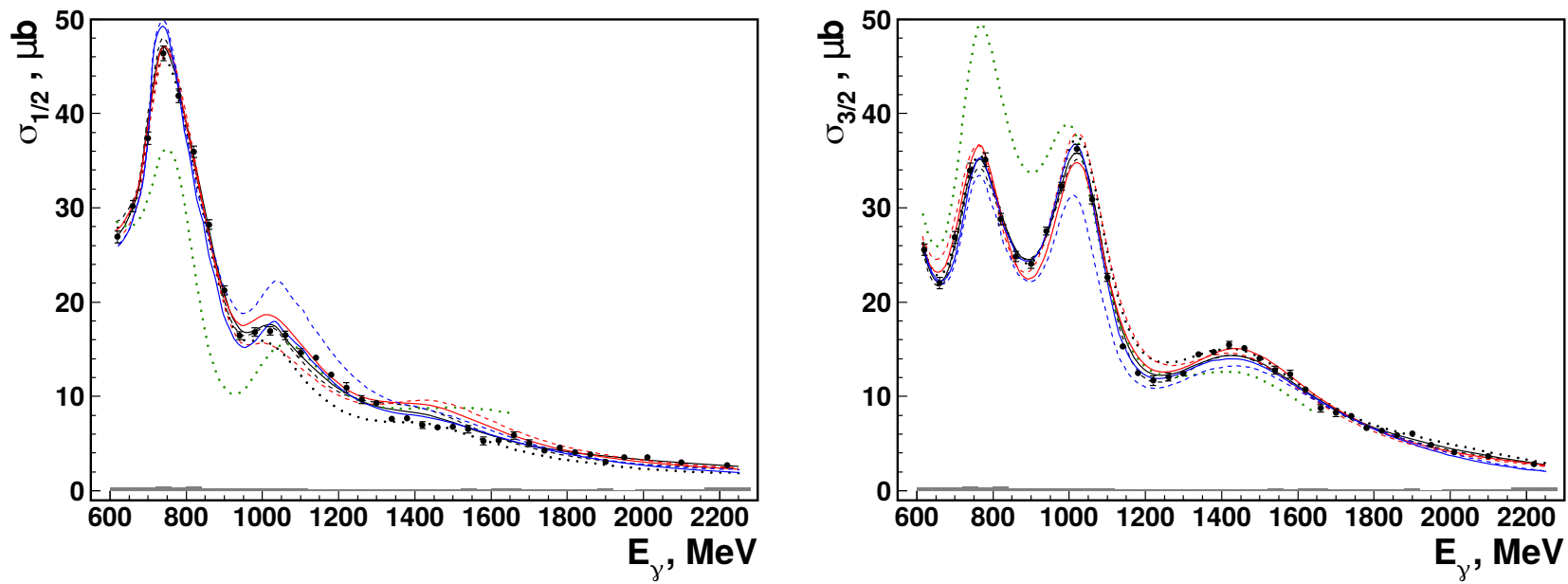

Figure 4. The spin dependent cross sections $\sigma_{1 / 2}$ (left) and $\sigma_{3 / 2}$ (right), which were extracted from the helicity asymmetry $E$ measurement, are plotted for the $p \pi^{0}$ final state (black points) together with different PWA solutions (solutions including this data: BnGa-201402 (black solid) [15], BnGa-2014-01 (black dashed) [41], SAID (PD03) [41] (red solid, curve calculated used solution PD03 for E and CM12 for the cross section), JüBo-2016-01 (blue solid) [41], and not including this data: BnGa-2011-02 (black dotted) [42], SAID CM12 (red dashed) [17], JüBo-2013-01 (blue dashed) [43], MAID2007 (green dotted) [18]). The systematic error is represented by the gray area. Taken from [9]. 


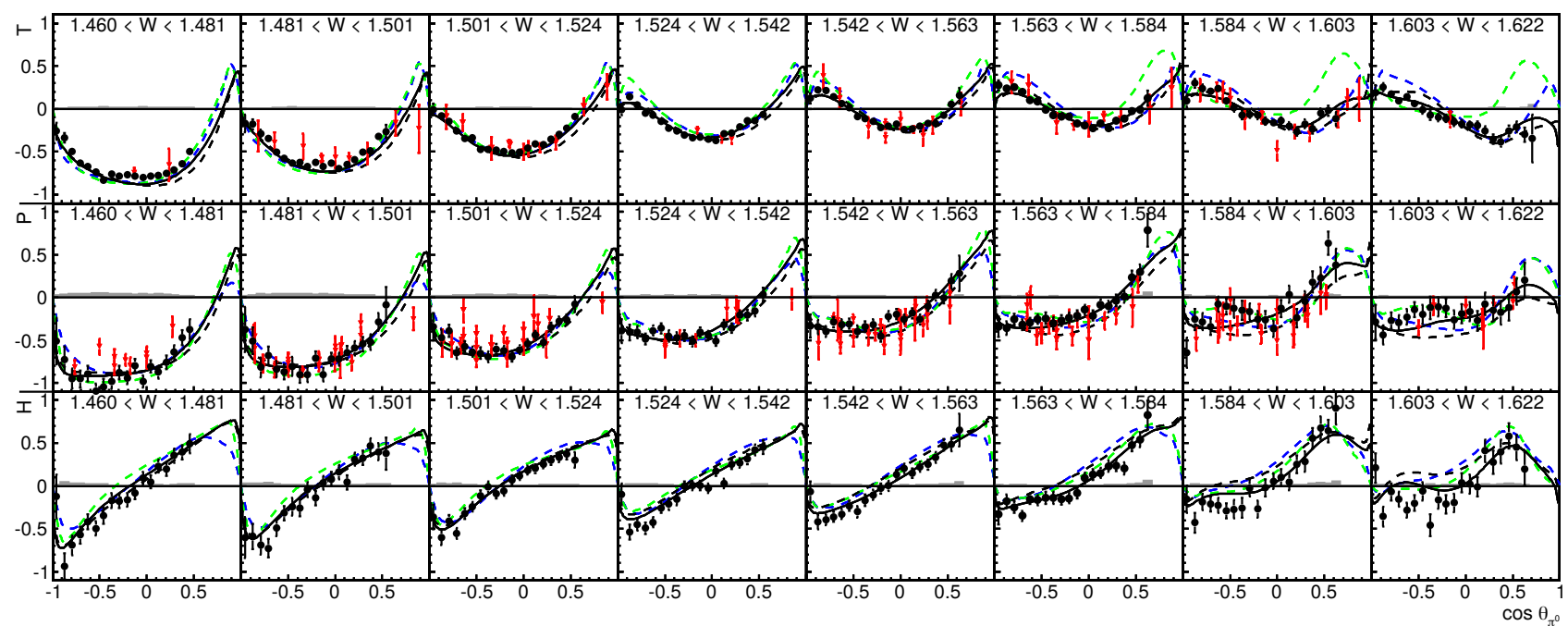

Figure 5. The results for the polarization observables $T, P$ and $H$ are shown for selected energy bins for the $p \pi^{0}$ final state (black points). The data is compared to previous measurements (red points) [44] and to different PWA predictions (dashed curves: BnGa-2011 (black) [42], MAID (green) [18], and SAID CM12 (blue) [17]) and PWA fit (BnGa-2014-02 (blak solid line) [15, 41]). Taken from [10].

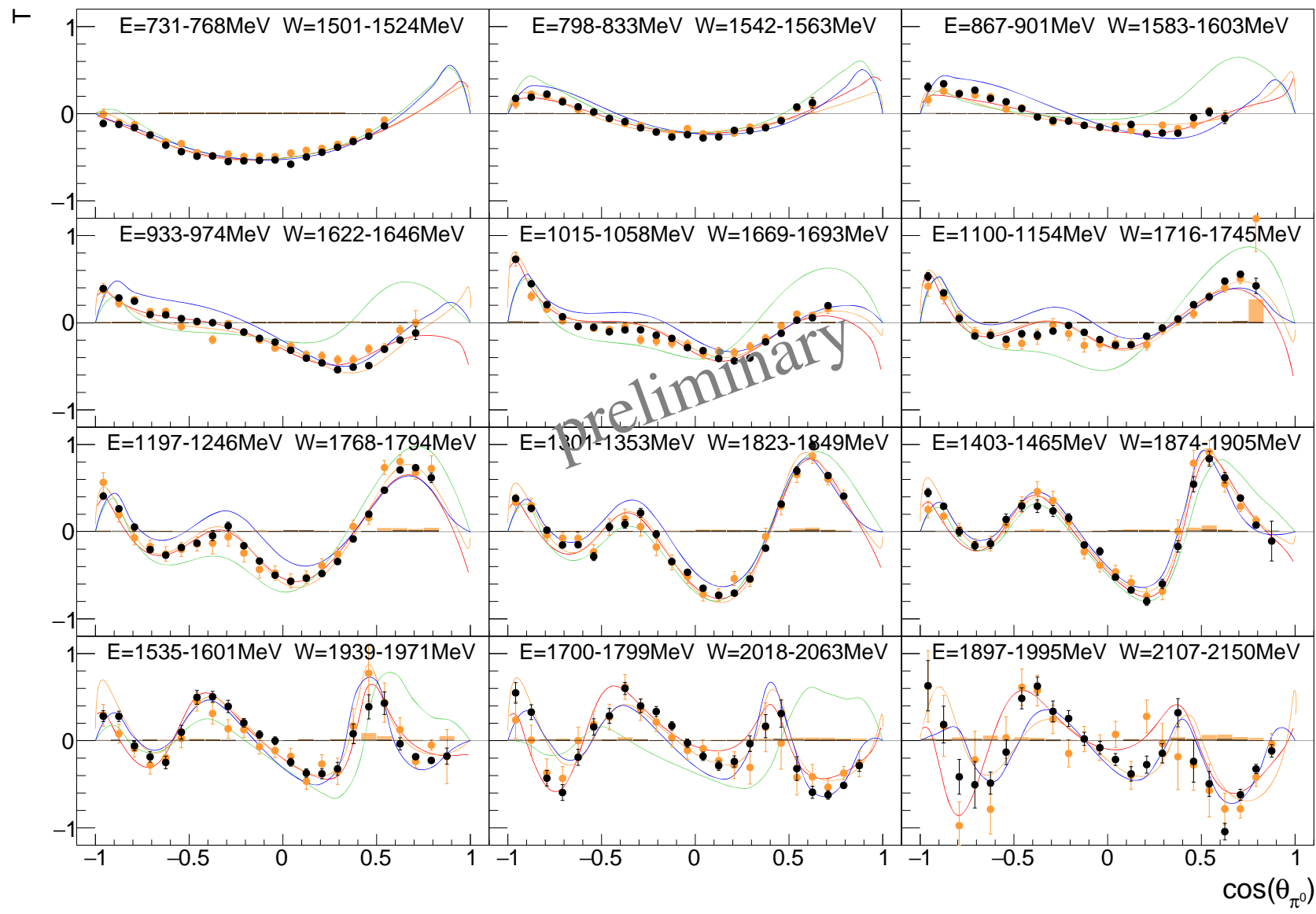

Figure 6. Preliminary results are shown for the polarization observable $T, P$ for the $p \pi^{0}$ final state. This new data (black points), which was taken after the CB upgrade from December 2017 to October 2018, is compared to already published data of the CBELSA/TAPS collaboration [10, 11]. In addition, different PWA curves are plotted as well (JüBo-2017 (orange line) [16], BnGa-2014-02 (red line) [15, 41], SAID-CM12 (blue line) [17] and MAID-2007 (green line) [18]). 


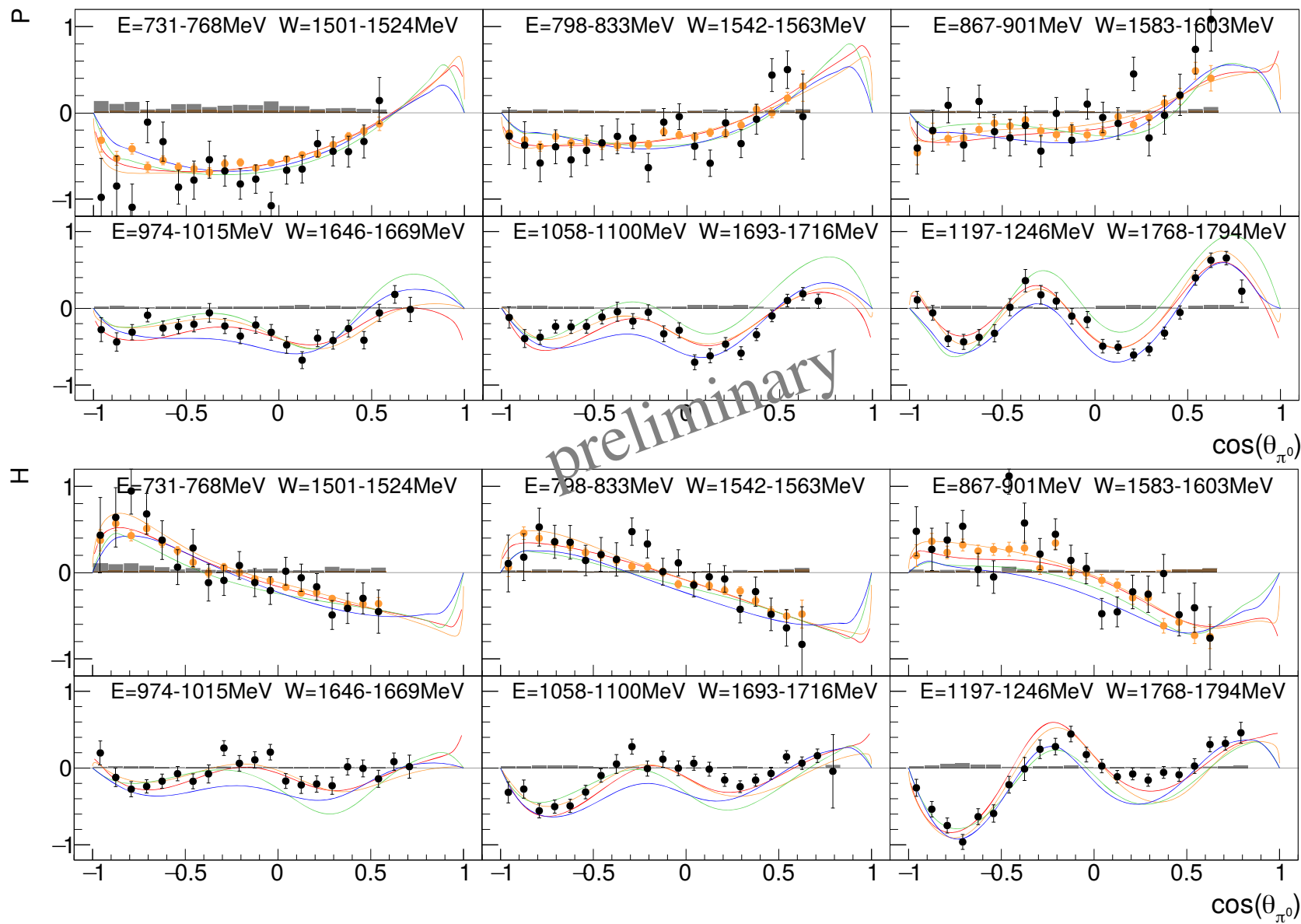

Figure 7. Preliminary results are shown for the polarization observables $P$ and $H$ for the $p \pi^{0}$ final state. This new data (black points), which was taken after the CB upgrade from December 2017 to October 2018, is compared to already published data of the CBELSA/TAPS collaboration [10, 11]. In addition, different PWA curves are plotted as well (JüBo-2017 (orange line) [16], BnGa2014-02 (red line) [15, 41], SAID-CM12 (blue line) [17] and MAID-2007 (green line) [18]).

body kinematics. Thus, more polarization observables than the ones listed in Table 1 become accessible [45]. The results for these observables are shown in references [29, 30]. Using this new data in their latest PWA, the $\mathrm{BnGa}$ group was able to determine the branching ratios of $N^{*}$ and $\Delta^{*}$ resonances into $N \pi, \Delta \pi, N(1440) \pi, N(1520) \pi, N(1535) \pi, N(1680) \pi$ or $N \sigma$ $[29,46]$. The extracted branching ratios show that, depending on the structure of the baryon wave function, some resonances prefer to decay into the ground state nucleons or deltas, while others decay with a significant branching ratio into excited nucleon or delta states $[29,30]$.

\subsection{Impact of polarization observables}

Using the determined double polarization observables $G, E, T, P$ and $H$ of the $p \pi^{0}$ final state by the CBELSA/TAPS collaboration, stronger constraints could be imposed on the photoproduction multipoles of the $p \pi^{0}$ final state by the BnGa, JüBo and SAID groups. As an example the real and imaginary parts of the $E_{0+}, E_{1+}, M_{1-}$ and $M_{2-}$ multipoles are shown in Figure 10. It becomes apparent that the fit error bars of the BnGa PWA decrease by a factor of 2.25 due to the inclusion of these new polarization measurements [10]. In addition, the deviation between the different PWA groups was investigated by taking the variance of all three PWA (BnGa, JüBo and SAID) summed over all $p \pi^{0}$ photoproduction multipoles up to $L=4$ [15]. Here, a clear reduction of the variance was observed due to inclusion of the new polarization data. Thus, the multipoles of the different PWA are slowly converging towards one solution.

Based on the latest polarization data from several collaborations, e.g. A2, CLAS, GRAAL, and CBELSA/TAPS, several resonances have been upgraded regarding their star-rating in the PDG [47].

\subsection{Summary and Outlook}

The CBELSA/TAPS collaboration has measured almost all of the polarization observables of the beam-target category for single and multi-meson final states with high precision, covering a large region in phase-space. The results were used by several PWA groups to constrain their solutions. A comparison of the multipoles shows that the new polarization data helps to reach the goal of 


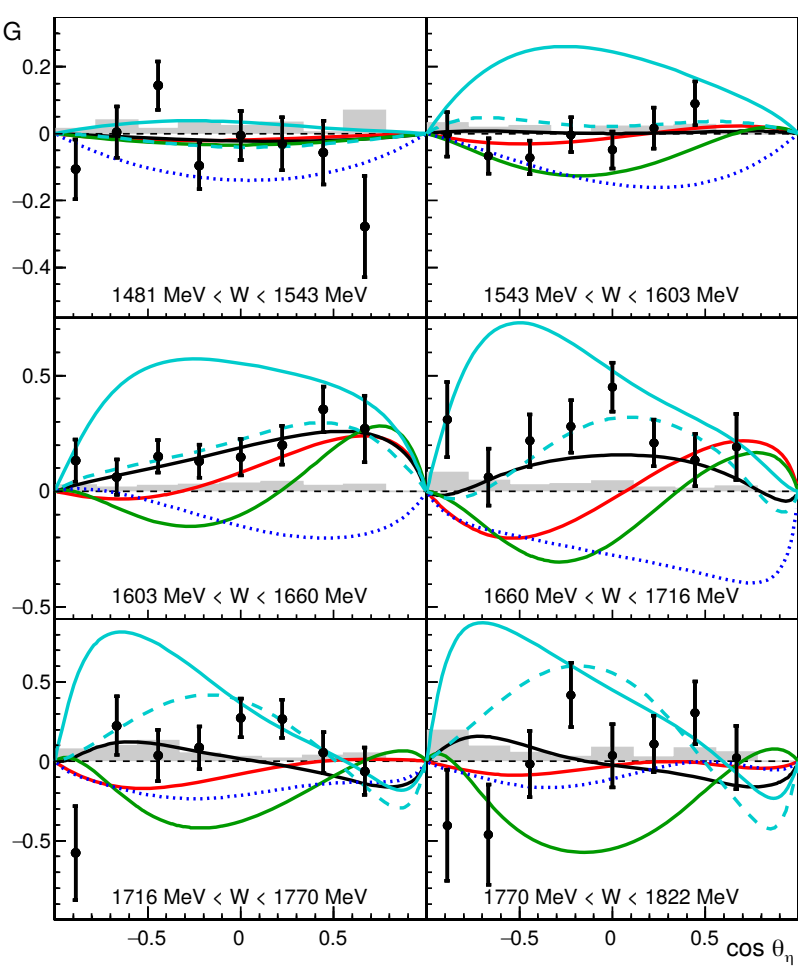

Figure 8. The double polarization observable $G$ is shown for the $p \eta$ final state (black points) together with different PWA solutions (latest fit of BnGa (black line) [40], BnGa2011-02 (red line) [42], $\eta$ MAID2018 (green line) [48], SAID-GE09 (dark blue dotted) [49], JüBo-2015 (light blue dashed line) [50] and JüBo2015-3 (light blue solid line) [51]). Taken from [14].

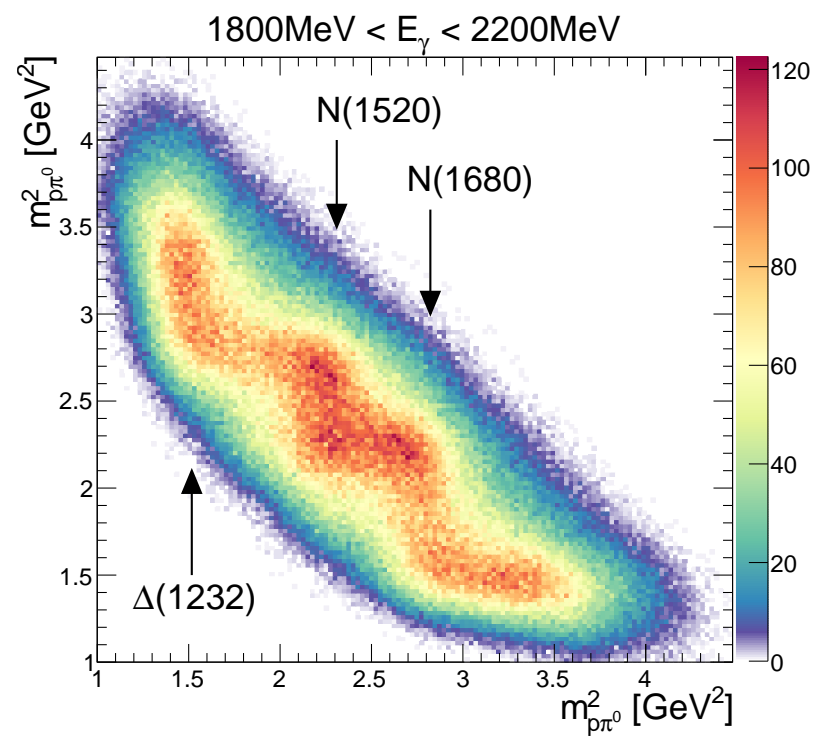

Figure 9. A Dalitz plot is depicted for the reaction $\gamma p \rightarrow p \pi^{0} \pi^{0}$ for an energy range of $1800 \mathrm{MeV}<E_{\gamma}<2200 \mathrm{MeV}$. The horizontal and vertical bands indicate the intermediate states.
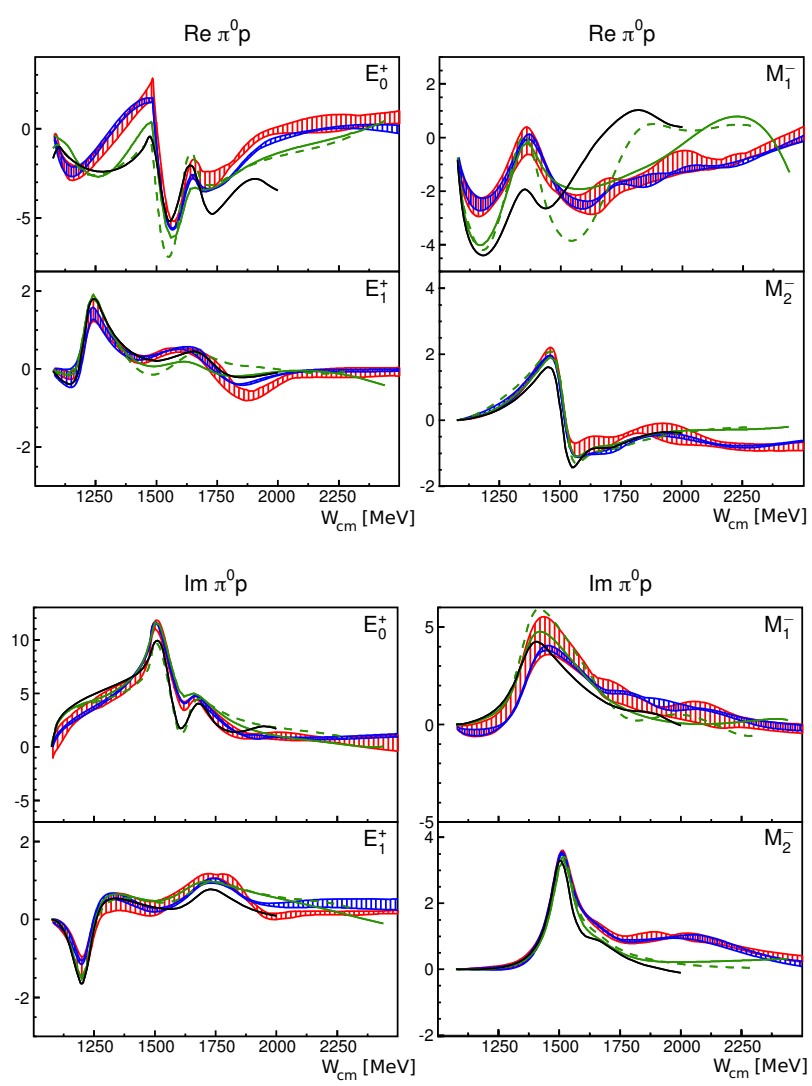

Figure 10. the real and imaginary parts of the $E_{0+}, E_{1+}, M_{1-}$ and $M_{2-}$ multipoles of the $p \pi^{0}$ final state are depicted. The red areas represent a variety of different fits of the BnGa2011-01 and BnGa2011-02 PWA [42], that do not include the new polarization data, while the blue areas represent the variety of solutions when including the new polarization data (BnGa-2014-02 PWA solution [15, 41]). In addition, the MAID (black line) [18], SAID-CM12 (solid green line) [17], SAID-SN11 (green dashed line) [52] and the JüBo-2015 (magenta line) [50] PWA fit solutions are shown as well. Taken from [11].

finding one unique solution. The results have now entered the PDG and the star-rating of several resonances was improved.

The CBELSA/TAPS experiment was successfully upgraded with a new readout electronics for the $\mathrm{CB}$ detector. The new data, that has been taken after the upgrade, show promising results with improved statistical precision. The finished detector upgrade allows to measure polarization observables for final states that involve neutral particles exclusively, with a high trigger efficiency. These final states, e.g. $n \pi^{0}$, are in particular important since there is only very few data available for these final states.

\section{References}

[1] U. Loring, B. C. Metsch and H. R. Petry, Eur. Phys. J. A 10, 395 (2001).

[2] R. G. Edwards, J. J. Dudek, D. G. Richards and S. J. Wallace, Phys. Rev. D 84, 074508 (2011).

[3] V. Burkert, these proceedings. 
[4] S. Strauch, these proceedings.

[5] M. Dugger et al. [CLAS Collaboration], Phys. Rev. C 88, (2013).

[6] S. Costanza, these proceedings.

[7] M. Sumihama et al. [LEPS Collaboration], Phy. Lett. B 657, 32 (2007).

[8] M. Gottschall et al. [CBELSA/TAPS Collaboration], Phys. Rev. Lett. 112, 012003 (2014).

[9] M. Gottschall et al. [CBELSA/TAPS Collaboration], arXiv:1904.12560v1 [nucl-ex] (2019).

[10] J. Hartmann et al. [CBELSA/TAPS Collaboration], Phys. Rev. Lett. 113, 062001 (2014).

[11] J. Hartmann et al. [CBELSA/TAPS Collaboration], Phys. Lett. B 748, 212 (2015).

[12] A. Thiel et al. [CBELSA/TAPS Collaboration], Phys. Rev. Lett. 109, 102001 (2012).

[13] A. Thiel et al. [CBELSA/TAPS Collaboration], Eur. Phys. J. A 53, 8 (2017).

[14] J. Müller et al. [CBELSA/TAPS Collaboration], Phys. Lett. B 803, 135323 (2020).

[15] A. V. Anisovich et al., Eur. Phys. J. A 52, 284 (2016).

[16] D. Rönchen, M. Döring and U.-G. Meißner, Eur. Phys. J. A 54110 (2018).

[17] R.L. Workman, et al., Phys. Rev. C 86, 015202 (2012).

[18] D. Drechsel, S.S. Kamalov, L. Tiator, Eur. Phys. J. A 34, 69 (2007).

[19] W. Hillert, Eur. Phys. J. A 28S1, 139 (2006).

[20] T. Jude, these proceedings.

[21] D. Elsner et al., Eur. Phys. J. A 39, 373 (2009).

[22] A. M. Sandorfi et al., J. Phys. G 38, 053001 (2011).

[23] E. Aker et al., Nucl. Instr. Meth. A 321, 69-108 (1992).

[24] Ch. Bradtke, H. Dutz, H. Peschel et al., Nucl. Instr. Meth. A 436, 430-442 (1999).

[25] S. Runkel, these proceedings.

[26] R. Novotny, IEEE Trans. Nucl. Sci. NS 38, 379-385 (1991).

[27] G. Suft et al., Nucl. Instr. Meth. A 531, 416-424 (2005).

[28] F. N. Afzal [CBELSA/TAPS Collaboration], EPJ Web Conf. 73, 04005 (2014).
[29] T. Seifen et al. [CBELSA/TAPS Collaboration], "Polarization observables in double neutral pion photoproduction", in preparation.

[30] T. Seifen [CBELSA/TAPS Collaboration], these proceedings.

[31] E. Gutz et al. [CBELSA/TAPS Collaboration], Eur. Phys. J. A 50, 74 (2014).

[32] H. Eberhardt et. al. [CBELSA/TAPS Collaboration], Phys. Lett. B 750, 453-458 (2015).

[33] C. Patrignani et al. (Particle Data Group), Chin. Phys. C 40, 100001 (2016) and 2017 update.

[34] Y. Wunderlich et al., Eur. Phys. J. A 53, no. 5, 86 (2017).

[35] A. V. Anisovich et. al., Phys. Lett. B 766, 357-361 (2017).

[36] B. Krusche et al., Prog. Part. Nucl. Phys. 51, 399 (2003).

[37] Y. Wunderlich, these proceedings.

[38] F. Afzal et al. [CBELSA/TAPS Collaboration], "Observation of the $\boldsymbol{p} \boldsymbol{\eta}^{\prime}$ cusp in the new precise beam asymmetry $\boldsymbol{\Sigma}$ data for $\boldsymbol{\gamma} \boldsymbol{p} \rightarrow \boldsymbol{p} \boldsymbol{\eta}^{\prime \prime}$, submitted to Phys. Rev. Lett.

[39] O. Bartalini et al. [GRAAL Collaboration], Eur. Phys. J. A 26, 399 (2005).

[40] A. Anisovich et al., Phys. Lett. B 785 626-630 (2018).

[41] V. Sokhoyan et al. [CBELSA/TAPS Collaboration], Eur.Phys. J. A 51, 95 (2015).

[42] A. V. Anisovich et al., Eur. Phys. J. A 48, 15 (2012).

[43] D. Rönchen et al., Eur. Phys. J. A 49, 44 (2013).

[44] W.J. Briscoe, D. Schott, I.I. Strakovsky, and R.L. Workman, GWU analysis center, gwdac.phys.gwu.edu/

[45] W. Roberts and T. Oed, Phys. Rev. C 71, 055201 (2005).

[46] A. Thiel et al., Phys. Rev. Lett. 114, 091803 (2015).

[47] M. Tanabashi et al. (Particle Data Group), Phys. Rev. D 98, 030001 (2018) and 2019 update.

[48] L. Tiator et al., Eur. Phys. J. A 54, 210 (2018).

[49] SAID: http://gwdac.phys.gwu.edu/

[50] D. Rönchen et al., Eur. Phys. J. A 5170 (2015).

[51] I. Senderovich et al., Phys. Lett. B 775, 64 (2016).

[52] R.L. Workman, et al., Phys. Rev. C 85, 025201 (2012) 